\title{
Linear detectors and precoding methods for massive MIMO
}

\section{Detectores lineares e métodos de pré-codificação para MIMO massivo}

\author{
Jean Marcel Faria Tonin ${ }^{1}$; Taufik Abrão ${ }^{2}$
}

\begin{abstract}
Detection in multiple-input-multiple-output (MIMO) wireless communication systems is a crucial procedure in receivers since the multiple access transmission schemes generate interference due to the simultaneous transmission along with the several antennas, unlike single-input-single-output (SISO) transmission schemes. Precoding is a technique in MIMO systems used to mitigate the effects of the channel over the received signal. Hence, it is possible to adjust continuously the transmitted information to reverse the effect of the wireless channel at the receiver side. In this work, linear sub-optimal detectors and precoders for massive MIMO (M-MIMO) systems are implemented, analyzed, and compared in terms of performance-complexity trade-off. It is also being considered numerical results in both channel scenarios: a) receiver and transmitter have perfect channel state information (CSI); b) complex channel coefficients are estimated with different levels of inaccuracy. Monte-Carlo simulations (MCS) reveal that linear zero-forcing (ZF) and minimum mean squared error (MMSE) massive MIMO detectors result in a certain robustness against multi-user interference when operating under low and medium system loading, $\mathscr{L}=\frac{K}{M}$, thanks to the favourable propagation phenomenon arising in massive MIMO systems.
\end{abstract}

Keywords: Detection. Precoding. Massive MIMO. Channel estimation. Wireless communications.

\section{Resumo}

A detecção em sistemas de comunicação sem fio de múltiplas entradas e saídas (MIMO) é um procedimento crucial em receptores, uma vez que os esquemas de transmissão de múltiplo acesso geram interferência devido à transmissão simultânea junto com as várias antenas, ao contrário de esquemas de transmissão de entrada única-saída única (SISO). A pré-codificação é uma técnica em sistemas MIMO usada para mitigar os efeitos do canal desvanecido sobre o sinal recebido. Portanto, é possível ajustar continuamente as informações transmitidas para reverter o efeito do canal sem fio no lado do receptor. Neste trabalho foram implementados, analisados e comparados detectores e métodos de pré-codificação lineares sub-ótimos para sistemas MIMO massivo (M-MIMO), sob a perspectiva do compromisso desempenho-complexidade. Foram considerados resultados numéricos em dois cenários de canal: a) o receptor e o transmissor têm informações perfeitas do estado de canal (CSI); b) os coeficientes complexos de canal são estimados com diferentes níveis de imprecisão. As simulações de Monte-Carlo (MCS) revelam certa robustez contra a interferência multiusuário para o detector linear de forçagem-zero $(\mathrm{ZF})$ e os detectores MIMO massivo baseados no mínimo erro quadrático médio (MMSE), operando sob carregamento de sistema baixo e médio, $\mathscr{L}=\frac{K}{M}$, graças ao fenômeno de propagação favorável que surge em sistemas MIMO massivo.

Palavras-chave: Detecção. Pré-codificação. MIMO massivo. Estimação de canal. Comunicação sem fio.

\footnotetext{
${ }^{1}$ Scientific Initiation Student, FA, Electrical Engineering Student, UEL, Londrina, Paraná, Brazil, E-mail: jean.faria.tonin@uel.br

${ }^{2} \mathrm{Ph}$. D. Department of Electrical Engineering, UEL, Londrina, Paraná, Brazil. E-mail: abrao@ ieee.org
} 


\section{Introduction}

Massive multiple-input-multiple-output (M-MIMO) has been of great interest in recent years, its ability to maximise the spectral/energy efficiency, increase the data rate and many other benefits has encouraged many researchers to study the extension of its benefits and, on the other hand, its negative points (MARZETTA et al., 2016). In this scenario, the large array gain, channel hardening and favorable propagation, among others, help to achieve better results in terms of quantity of information transmitted, and, just as important, in terms of how much of this information is successfully received (MARINELLO; ABRAO, 2014).

Combiners were largely exploited in MIMO systems, (COSTA; MUSSI; ABRAO, 2016), and are of great interest in M-MIMO systems, mainly ones that are linear, such as the matched filter (MF) studied in (LIANG; ZHANG; SHEN, 2015) as well as the zero forcing (ZF), which is also studied in (YOUNAS et al., 2017), and the one based on minimum mean squared error (MMSE) (BOROUJERDI; HAGHIGHATSHOAR; CAIRE, 2018).

MF, as known as maximal-ratio combining (MRC), is the most simple receiver studied in this paper, but has a good trade off between complexity and performance, especially as the number of antennas grows, however, it is the only method here that features a bit error rate (BER) floor. ZF is a popular choice due to its optimal performance in multiple scenarios allied with a relative low complexity, although it still requires matrix inversion operation. The MMSE, sometimes referred as regularized zero forcing (RZF), performs very well in scenarios limited by noise, but it is relatively computationally costly in M-MIMO scenarios when compared with $\mathrm{ZF}$ and MF counterparts.

Linear precoding (LP) and combiners are a very popular choice due to its relatively low complexity allied to good performance. The LP methods analyzed herein include the MF and the ZF (WIESEL; ELDAR; SHAMAI, 2008) in their precoding versions, and the singular-value decomposition (SVD) precoder (BUSCHE; VANAEV; ROHLING, 2009) is also studied, although it is very different from the other two methods explained.

Both MF and ZF precoders have, roughly, the same characteristics of their combiner versions, although it has the benefit of multiplying only the signal vector, preventing from amplifying the noise, which happens in scenarios where only combiners are employed.
SVD, on the other hand, has a very distinct characteristic, as it requires processing both at the base station (BS) and at the user terminal, depending on the case applied this can be either beneficial or harmful, since it takes some of the responsibility from the $\mathrm{BS}$ and gives it to the user.

Precoders have a huge importance in wireless transmissions, mostly in a downlink (DL) direction. This paper is also studying its performance in multiple access scenarios, modifying parameters such as the number of users accessing the system simultaneously, and the number of BS antennas.

Motivated by the benefits of M-MIMO systems listed above (BJÖRNSON; HOYDIS; SANGUINETTI, 2018), this work aims to recreate results in the literature and expand the scenario varying the channel and system parameters aiming to construct and evaluate an extensive system configuration in terms of bit rrror rate.

Notation: Bold lower case letters represent vectors, meanwhile bold upper case letters denote matrices. The operators $(\cdot)^{T},(\cdot)^{H}$ and $\mathbb{E}\{\cdot\}$ denote the transpose, the conjugate transpose and the expected value, respectively. The $M \times M$ identity matrix is represented by $I_{M} \cdot\|\cdot\|$ stands for the euclidian norm, as known as, the $l_{2}$ norm. $\mathbf{0}_{M}$ is a vector with $\mathrm{M}$ columns in which all elements are the number zero. A random variable $n \sim$ $\mathscr{C} \mathscr{N}\left(m, \sigma^{2}\right)$ is complex Gaussian distributed with mean value $m$ and variance $\sigma^{2}$. $\mathbf{U} \in \mathbb{C}^{K \times M}$ denotes a matrix with $\mathrm{K}$ rows and $\mathrm{M}$ columns and its elements are all complex-valued.

\section{System model}

\section{$\operatorname{Uplink}(U L)$}

Let $\mathbf{x}_{U L}=\left(x_{1}, \ldots, x_{K}\right)^{T}$ be the transmitted symbols vector, where $K$ is the number of users and $M$ is the number of antennas at the BS, it is also defined $\mathbf{H}_{U L} \in$ $\mathbb{C}^{M \times K}$ as the channel coefficient matrix, which is statistically distributed as $h_{m, k} \sim \mathscr{C} \mathscr{N}(0,1)$. At the BS, the receiving vector in a symbol period interval is given by equation (1):

$$
\mathbf{y}_{U L}=\mathbf{H}_{U L} \mathbf{x}_{U L}+\mathbf{n}_{U L},
$$

where $\mathbf{n}_{U L} \in \mathbb{C}^{M \times 1}$ is a zero mean Gaussian noise vector, and $\mathbb{E}\left\{\mathbf{n}_{U L} \mathbf{n}_{U L}{ }^{H}\right\}=\sigma^{2} \mathbf{I}_{M}$, where $\sigma^{2}$ is the noise variance in each received antenna. 


\section{Downlink $(D L)$}

Defining $n_{s}$ as the number of information symbols transmitted per channel $\left(n_{s} \leq \min (M, K)\right)$, and $\mathbf{z}=\left(z_{1}, \ldots, z_{n_{s}}\right)^{T} \in \mathbb{C}^{n_{s}}$ the data symbol vector, and also the $M \times n_{s}$, precoding matrix $\mathbf{T}$, which is applied to the data symbol vector to produce the transmitted vector defined in equation (2):

$$
\mathbf{x}_{D L}=\mathbf{T z}
$$

The receiving vector $\mathbf{y}$ at the user equipment (UE) is then given by equation (3):

$$
\mathbf{y}_{D L}=\mathbf{H}_{D L} \mathbf{x}_{D L}+\mathbf{n}_{D L}=\mathbf{H}_{D L} \mathbf{T} \mathbf{z}+\mathbf{n}_{D L} .
$$

Nonetheless, the channel matrix and the noise vector modify their dimensions when compared to the UL model, now $\mathbf{H}_{D L} \in \mathbb{C}^{K \times M}$, since it is the conjugate of the Uplink Channel Matrix, and $\mathbf{n}_{D L} \in \mathbb{C}^{K}$.

\section{Linear detection and precoding for M-MIMO}

Detectors and precoders are essential steps in a communication system, in order to reduce the complexity at the UE, it is preferable to execute the majority of signal processing at the BS. In this context, precoding methods are very useful in DL scenarios; on the other hand, combiners are important in UL direction as part of detection process of $K$ UE symbol information. Figure 1(a) illustrates the DL precoding under $M$ BS antennas, meanwhile Figure 1(b) depicts UL combiners processes serving $K$ user equipments.

\section{Massive MIMO systems}

A massive MIMO (M-MIMO) system (MARZETTA et al., 2016) represents a cellular network with a large number of antennas at the BS, $M>>1$, usually $M>100$, but $M>64$ is already enough to see the benefits of MMIMO, in terms of favorable propagation and channel hardening, and a number of users $K$ such that the load factor $\beta=\frac{K}{M}<1$, usually $\beta<0.25$ in classical M-MIMO system scenarios.

The canonical M-MIMO assumes a space-division multiple access (SDMA) transmission structure (BJÖRNSON; HOYDIS; SANGUINETTI, 2017). It allows the system to increase the overall system spectral efficiency (SE) and communicate with multiple users simultaneously. It also uses time-division duplexing (TDD) operation, in which the BS estimates the channel from pilots sent by the users, and since the channel is reciprocal, this estimation can be used such in UL and DL transmissions.

Two crucial characteristics of M-MIMO systems are channel hardening and favorable propagation effects, defined as follows.

Channel hardening: when $M \rightarrow \infty$, the fading channel behaves as if it was deterministic. This happens because, due to the law of large numbers, the propagation channel is close to its expected value, which can be seen in equation (4):

$$
\frac{\left\|\mathbf{h}_{k}\right\|^{2}}{\mathbb{E}\left\{\left\|\mathbf{h}_{k}\right\|^{2}\right\}} \rightarrow 1
$$

where $\mathbf{h}$ is the row vector of the Channel Matrix relative to each user, which is identified by the index $k$.

Favorable propagation. Again, when $M \rightarrow \infty$, the directions of two users are asymptotically orthogonal, which facilitates the interference mitigation among the UEs. The same can be visualized in equation (5)

$$
\frac{\left(\mathbf{h}_{i}\right)^{H} \mathbf{h}_{k}}{\mathbb{E}\left\{|| \mathbf{h}_{i} \|^{2}\right\} \mathbb{E}\left\{\left\|\mathbf{h}_{k}\right\|^{2}\right\}} \rightarrow 0,
$$

where indexes $k$ and $i$ identify the users

Such characteristics listed above, along with the large array gain confer a superior performance to the M-MIMO when compared to the conventional MIMO system.

\section{Linear detectors for M-MIMO}

Linear detectors (LD) estimate the information transmitted through a linear transformation of the received signal, this generates a low complexity detection, but with a lower performance (ZU, 2013). In general, linear detectors are defined as in equation (6):

$$
\hat{\mathbf{x}}=f\left(\mathbf{Q y}_{U L}\right),
$$

where $\mathbf{Q}$ is the transformation matrix and $f($.$) is a slicer$ that approximates $\mathbf{Q} \mathbf{y}_{U L}$, based on the Euclidean distance, to the nearest symbol in the modulation alphabet in order to obtain $\hat{\mathbf{x}}$.

\section{MF detector}

Matched Filter (MF) is one of the simplest linear detectors, it maximizes SNR and treats interference between antennas as simple noise (CHOCKALINGAM; RANJAN, 2014). 
Figure 1 - Massive MIMO communications: (a) Downlink and (b) Uplink.

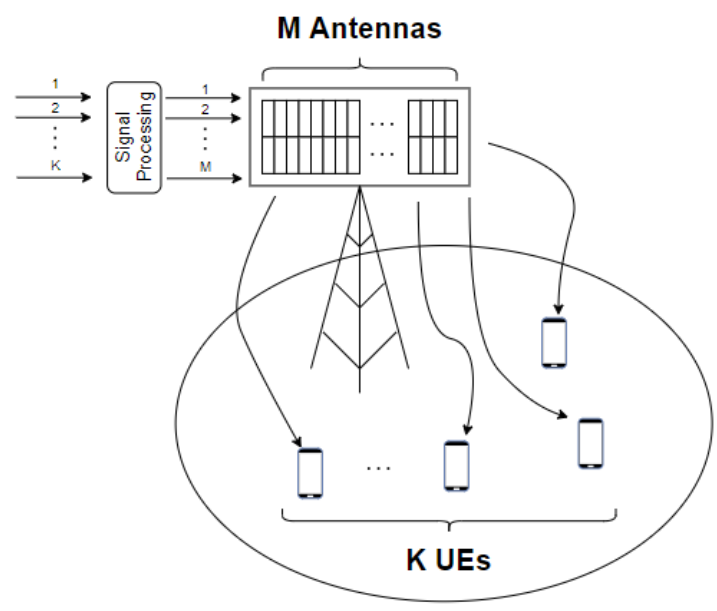

Source: The authors.

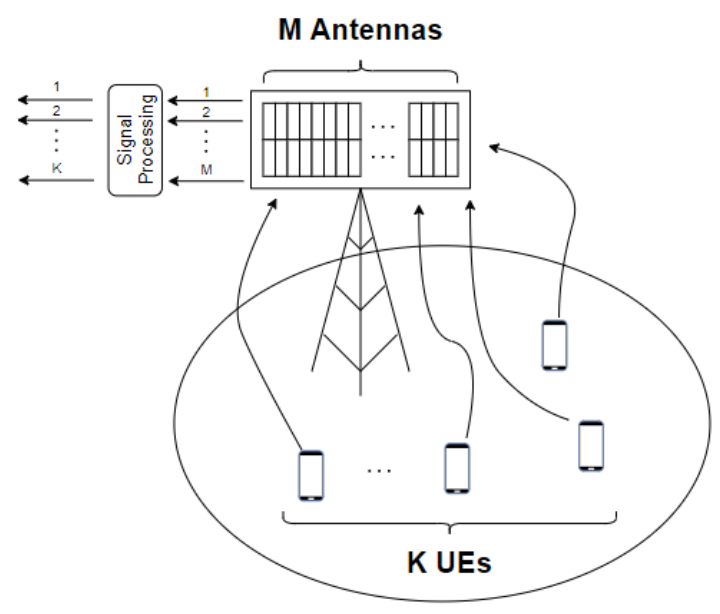

(b)
Let $\mathbf{H}_{U L}$ be the channel gain matrix, the received signal vector $\mathbf{y}$ is given by:

$$
\begin{array}{r}
\mathbf{y}_{U L}=\mathbf{H}_{U L} \mathbf{x}_{U L}+\mathbf{n}_{U L}=\sum_{i=1}^{K} \mathbf{h}_{i} x_{i}+\mathbf{n}_{U L} \\
=\mathbf{h}_{k} x_{k}+\sum_{i=1, i \neq k}^{K} \mathbf{h}_{i} x_{i}+\mathbf{n}_{U L} .
\end{array}
$$

The first term in (7) is the desired signal and the second is the interference from the other antennas. MF simply considers the second term as noise and obtains an estimate given by equation (8):

$$
\tilde{x}_{k}=\frac{\mathbf{h}_{k}^{*}}{\left\|\mathbf{h}_{k}\right\|^{2}} \mathbf{y}_{U L},
$$

$\hat{\mathbf{x}}$ can be estimated using a slicer. Therefore, the $k$-th row $\left(\mathbf{q}_{k}^{M F}\right)$ of $\mathbf{Q}_{\mathrm{MF}}$ is given by equation (9)

$$
\mathbf{q}_{k}^{M F}=\frac{\mathbf{h}_{k}^{*}}{\left\|\mathbf{h}_{k}\right\|^{2}},
$$

and $\mathbf{Q}_{\mathrm{MF}}=\left[\mathbf{q}_{1}^{M F} ; \mathbf{q}_{2}^{M F} ; \cdots ; \mathbf{q}_{K}^{M F}\right]$. It is possible to write it in vector form as in equation (10):

$$
\tilde{\mathbf{x}}_{\mathrm{MF}}=\mathbf{Q}_{\mathrm{MF}} \mathbf{y}_{U L}
$$

\section{ZF detector}

Zero forcing $(\mathrm{ZF})$ is based on canceling all interference using the pseudo-inverse of the channel matrix $\mathbf{H}_{U L}$.

Equation (11) defines $\mathbf{Q}_{\mathrm{ZF}}$, the $M \times n_{s}$ pseudo-inverse of $\mathbf{H}_{U L}$ :

$$
\mathbf{Q}_{\mathrm{ZF}}=\left(\mathbf{H}_{U L}{ }^{H} \mathbf{H}_{U L}\right)^{-1} \mathbf{H}_{U L}{ }^{H},
$$

$\mathbf{q}_{k}$ is defined as the $k$ th row of $\mathbf{Q}_{\mathrm{ZF}}$, when applied to $\mathbf{y}$, it is obtained as in equation (12):

$$
\begin{array}{r}
\tilde{x}_{k}=\mathbf{q}_{k} \mathbf{y}=\mathbf{q}_{k} \mathbf{H} \mathbf{x}_{U L}+\mathbf{q}_{k} \mathbf{n}_{U L} \\
=x_{k}+\mathbf{q}_{k} \mathbf{n}_{U L} .
\end{array}
$$

Since $\mathbf{Q}_{\mathrm{ZF}} \mathbf{H}_{U L}=\mathbf{I}_{K}$, the interference is completely canceled, however, the noise $\mathbf{n}_{U L}$ is amplified by $\mathbf{q}_{k}$, and the SNR is given by equation (13):

$$
S N R_{k}=\frac{\left|x_{k}\right|^{2}}{\left\|\mathbf{q}_{k}\right\|^{2} \sigma^{2}} .
$$

In vector form, the $\mathrm{ZF}$ solution is given by equation (14):

$$
\tilde{\mathbf{x}}_{\mathrm{ZF}}=\mathbf{Q}_{\mathrm{ZF}} \mathbf{y}_{U L}
$$

\section{MMSE detector}

The minimum mean squared error (MMSE) detector is named due to its transformation matrix, which aims to minimize the mean square error between the transmit vector and the estimated vector. The transformation matrix $\mathbf{Q}_{\text {Mmse }}$ is given by equation (15):

$$
\mathbf{Q}_{\mathrm{MMSE}}=\left(\mathbf{H}_{U L}{ }^{H} \mathbf{H}_{U L}+\sigma^{2} \mathbf{I}_{K}\right)^{-1} \mathbf{H}_{U L}{ }^{H},
$$

where $\sigma^{2}$ is the noise variance, and the MMSE data estimation is given by equation (16):

$$
\tilde{\mathbf{x}}_{\mathrm{MMSE}}=\mathbf{Q}_{\mathrm{MMSE}} \mathbf{y}_{U L}
$$


MMSE combines the best aspects of ZF and MF detectors, at high SNR $(\sigma \rightarrow 0)$, the performance is similar to the zero forcing detector. At low SNR $(\sigma \rightarrow \infty)$ it behaves like MF, because of the prominence of the diagonal entries of $\mathbf{H}_{U L}{ }^{H} \mathbf{H}_{U L}+\sigma^{2} \mathbf{I}_{K}$. MMSE performs better than MF and ZF over the entire range of SNRs, however it requires knowledge of $\sigma^{2}$, which MF and ZF do not need.

\section{Precoding for M-MIMO}

Precoding is based on treating the signal before it is sent, to reverse the negative effects of the channel on the received signal. This treatment is done by a precoding matrix that is multiplied to the vector of transmitted signals, as in equation (2). Most precoders do not require the use of elaborated combiner at the receiver, which reduces the complexity of receiving and processing the signal at the mobile UE. In this case, only a slicer would be demanded to approach the received signal to the closest symbol in the constellation based on the Euclidean distance decision.

\section{$M F$ precoding}

This precoding method resembles MF detector, as it uses the hermitian of the channel gain matrix $\left(\mathbf{T}_{\mathrm{MF}}\right)$ to cancel the channel interference, as shown in equation (17)

$$
\mathbf{T}_{\mathrm{MF}}=\mathbf{H}_{D L}{ }^{H} .
$$

However this precoding method does not multiply the noise vector by $\mathbf{T}_{\mathrm{MF}}$, as the MF detector does, instead only the signal vector is multiplied, which can be seen in equation (18), resulting in a maximized SNR

$$
\mathbf{y}_{D L}=\mathbf{H}_{D L} \mathbf{T}_{\mathrm{MF}} \mathbf{z}+\mathbf{n}_{D L}
$$

\section{$Z F$ precoding}

Similarly to zero forcing detector, this method tries to cancel all the interference of the channel in the transmitted symbol vector $(\mathbf{z})$. However, in the precoding form it multiplies $\mathbf{z}$ by the pseudo-inverse of $\mathbf{H}_{D L}$, preceding the effects of the channel. The precoding matrix $\left(\mathbf{T}_{\mathrm{ZF}}\right)$ is given by equation (19):

$$
\mathbf{T}_{\mathrm{ZF}}=\mathbf{H}_{D L}{ }^{H}\left(\mathbf{H}_{D L} \cdot \mathbf{H}_{D L}{ }^{H}\right)^{-1} .
$$

Then it is applied to the transmitted vector, as shown in equation (20):

$$
\mathbf{y}_{D L}=\mathbf{H}_{D L} \mathbf{T}_{\mathrm{ZF}} \mathbf{z}+\mathbf{n}_{D L}
$$

In the receptor, no equalization method is necessary once the matrix $\mathbf{T}_{\mathrm{ZF}}$ already cancels the effect of the channel. However it is necessary to send information about the channel back to the transmitter to make feasible the calculation of $\mathbf{T}_{\mathrm{ZF}}$.

\section{SVD precoding}

A well-known precoding technique is the singular value decomposition (SVD), it consists of decomposing each value of the channel matrix, transforming the channel into multiple parallel subchannels, which ends up reducing the detection complexity. This method's main limitation is that it requires knowledge of the channel in both the transmitter and the receiver.

The SVD of the channel matrix $\mathbf{H}_{D L}$ is given by equation (21):

$$
\mathbf{H}_{D L}=\mathbf{U} \Lambda \mathbf{V}^{H},
$$

where $\mathbf{U} \in \mathbb{C}^{K \times K}, \Lambda \in \mathbb{C}^{K \times K}, \mathbf{V} \in \mathbb{C}^{M \times M}$, such that $\mathbf{U} \mathbf{U}^{H}=\mathbf{I}_{K}, \mathbf{V} \mathbf{V}^{H}=\mathbf{I}_{M}$ and $\Lambda=\operatorname{diag}\left(\lambda_{1}, \ldots, \lambda_{K}\right)$ are the singular values of $\mathbf{H}_{D L}$, with $\lambda_{1} \geq \lambda_{2} \geq \ldots \geq \lambda_{K} \geq 0$. $\widetilde{\mathbf{V}}$ $\in \mathbb{C}^{M \times n_{s}}$, is defined as the submatrix with the first $n_{s}$ columns of $\mathbf{V}$. SVD uses $\mathbf{T}_{\mathrm{SVD}}$, defined in equation (22):

$$
\mathbf{T}_{\mathrm{SVD}}=\tilde{\mathbf{V}}
$$

The received vector $\mathbf{y}_{D L}$ is, then, given by equation (23):

$$
\mathbf{y}_{D L}=\mathbf{H}_{D L} \mathbf{T}_{\mathrm{SVD}} \mathbf{u}+\mathbf{n}_{D L}
$$

The receiver computes:

$$
\mathbf{r}=\mathbf{U}^{H} \mathbf{y}_{D L}=\tilde{\Lambda} \mathbf{u}+\mathbf{w}
$$

where $\mathbf{w} \in \mathbb{C}^{n_{s}}$ is a Gaussian noise, $\tilde{\Lambda}=\operatorname{diag}\left(\lambda_{1}, \ldots \lambda_{n_{s}}\right)$, $\mathbf{r}=\left(r_{1}, \ldots, r_{n_{s}}\right)^{T}$ in equation (24). Therefore, SVD transforms the channel in $n_{s}$ parallel subchannels, as shown in equation (25):

$$
r_{i}=\lambda_{i} u_{i}+w_{i}, i=1, \ldots, n_{s}
$$

A drawback of the SVD precoder is that it is necessary to have information about the channel at both the receiver and at the transmitter sides to enable the task of multiplying the signal vector by $\mathbf{T}_{\mathrm{SVD}}$, and, simultaneously, multiplying the received vector by $\mathbf{U}^{H}$. 


\section{Numerical results}

Extensive numerical analyses have been carried out using MatLab mathematical simulations. It was possible to compare linear detectors, including MF, ZF and MMSE, with precoding methods, namely MF, ZF and SVD. The main parameter values deployed in the Monte-Carlo simulations (MCS) are listed in Table 1. The non-light-of-sight (NLOS) fading channel for the uplink scenario is generated statistically as

$$
\mathbf{H}_{U L}=\sqrt{0.5} \cdot \operatorname{randn}(M, K)+j \sqrt{0.5} \cdot \operatorname{randn}(M, K),
$$

where $\operatorname{randn}(M, K)$ represents a $M \times K$ matrix which elements following a Gaussian distribuition $\mathscr{N}(0,1)$. In the downlink scenario, the same channel model is adopted, however its dimensions are transposed, obtaining a $K \times M$ matrix.

Table 1 - Simulation Parameter Values

\begin{tabular}{ll}
\hline \multicolumn{2}{c}{ Massive MIMO scenario } \\
\hline \# UEs & $K \in\{1,2,4,8,16\}$ users \\
\# BS antennas & $M \in\{64,128,256\}$ \\
System Loading & $\mathscr{L}=\frac{K}{M} \in\{0.02 ; 0.25\}$ \\
\# UE antennas & 1 antenna/UE \\
SNR & $\gamma \in\{-10: 2: 10\}$ dB \\
\# parallel channels (SVD) & $n_{S}=\min (M, K)$ \\
Channel fading & Rayleigh, $\mathbf{H}$ \\
\hline
\end{tabular}

Source: The authors.

\section{Detection analysis under perfect CSI}

In this section the performance of detectors considering an UL M-MIMO scenario was analyzed. The modulation used is 16-QAM. In order to study the effects of the variation of the number of users $K$ and the number of antennas $M$ at the BS, over the bit-error-rate (BER), Figure 2 depicts Monte-Carlo numerical simulation $(\mathrm{MCS})$ results for BER $\times$ SNR considering different $M$ and $K$ values: Figure 2(a) considers $K=16$ and $M=64$, Figure 2(b) considers $K=8$ and $M=128$, Figure 2(c) considers $K=4$ and $M=256$.

To investigate the effect of system loading on the BER performance, Figure 3 depicts MCS results for BER $\times$ system loading, $\mathscr{L}=\frac{K}{M} \in\{0.02 ; 0.25\}$. As one can see, the performance of all combiners over 16-QAM is improved when the loading factor $\mathscr{L}$ decreases. As expected the BER performance is also improved when the UL MMIMO system operates under medium or high SNR scenarios, simulated in Figure 3(b), when compared to the low SNR scenario in Figure 3(a).

\section{Detection analysis under CSI errors}

The adopted channel error estimates model is defined by equation (27):

$$
\widehat{\mathbf{H}}=\sqrt{1-\tau^{2}} \cdot \mathbf{H}+\tau \cdot \mathbf{V}
$$

where matrices $\mathbf{H}$ and $\mathbf{V}$ are independent identically distributed (i.i.d.) complex Gaussian random variables with zero mean and unit variance, computed as in equation (26), $\mathbf{H}$ and $\widehat{\mathbf{H}}$ are not specified because they are valid for both UL and DL cases.. In addition, the accuracy estimation parameter $\tau \in[0,1]$ is applied to reflect the accuracy of the channel estimates, e.g., $\tau=0$ represents the perfect channel estimation, $\widehat{\mathbf{H}} \equiv \mathbf{H}$, whereas $\tau=1$ corresponds to the case that the channel estimate is completely uncorrelated with the actual channel response (MI et al., 2017).

Figures 4 and 6 depict the BER performance $\times$ SNR with $\tau=0.2$ and $\tau=0.4$, respectively, in which a) represents $K=16$ and $M=64$, b) $K=8$ and $M=128$ and c) $K=4$ and $M=256$. Such graphics facilitate the analysis with a larger set of SNR values. Meanwhile, Figures 5 and 7 also study the scenarios with $\tau=0.2$ and $\tau=0.4$, respectively; however, such results explore system loading variations operating under a) $S N R=-4 d B$ and b) $S N R=+2 d B$. Comparing with perfect CSI estimates $(\tau=0)$, one can infer that for the parameter $\tau=0.2$, the BER performance degradation becomes remarkable for $\mathscr{L} \geq 0.250$, i.e., under such condition, BER floor arises in all the three linear M-MIMO detectors when system loading (multiuser interference) increases. Such situation becomes more dramatic for higher error estimates, i.e. $\tau=0.4$, when a BER floor becomes apparent when $\mathscr{L} \geq 0.0625$.

\section{Precoding analysis under perfect CSI}

To analyze the downlink M-MIMO performance, it was considered a 4-QAM modulation while the number of users $K$, the number of antennas $M$, and the SNR was varied. Figure 8 depicts the results obtained, with $K=16$ and $M=64$ in a) and $K=8$ and $M=128$ in b). It is possible to see that all precoders improve their results for medium and high SNR, although MF reaches its BER floor, ZF and SVD manage to achieve optimal results. When the load factor $\mathscr{L}$ decreases, the performance is improved, as one can see in Figure 9. Results for higher system loading and SNR values are optimal and even achieve zero bit error rate. 
Figure 2 - MCS result for the detectors analysis with perfect CSI, different number of users $(K)$, and number of BS antennas (M): (a) $K=16$ and $M=64$; (b) $K=8$ and $M=128$ and (c) $K=4$ and $M=256$.

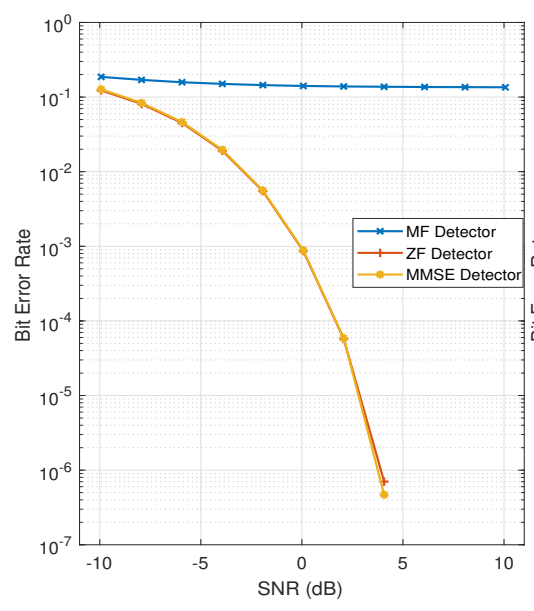

Source: The authors

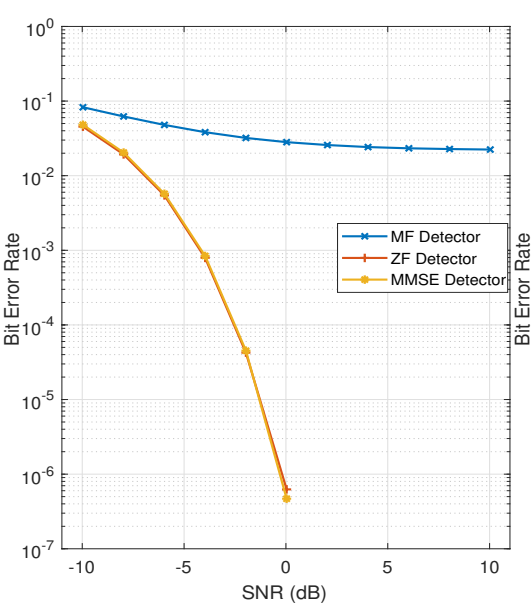

(b)

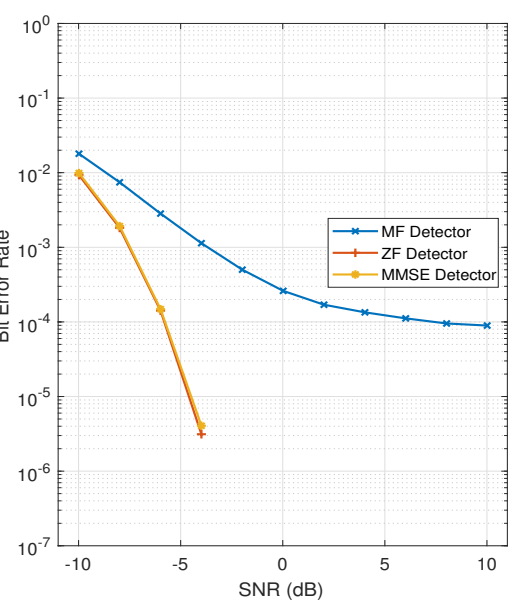

(c)

Figure 3 - MCS results for the detectors analysis for the average BER $\times$ system loading $\mathscr{L}$, considering perfect CSI estimates and $M=64 \mathrm{BS}$ antennas under low and medium SNR regime: (a) $\mathrm{SNR}=-4 \mathrm{~dB}$ and (b) $\mathrm{SNR}=+2 \mathrm{~dB}$.

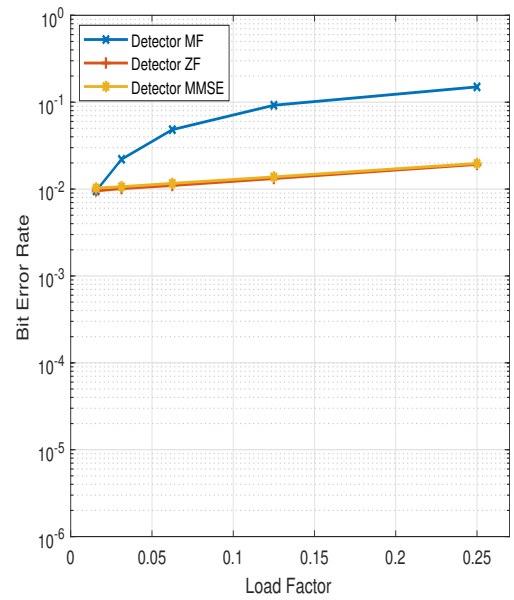

(a)

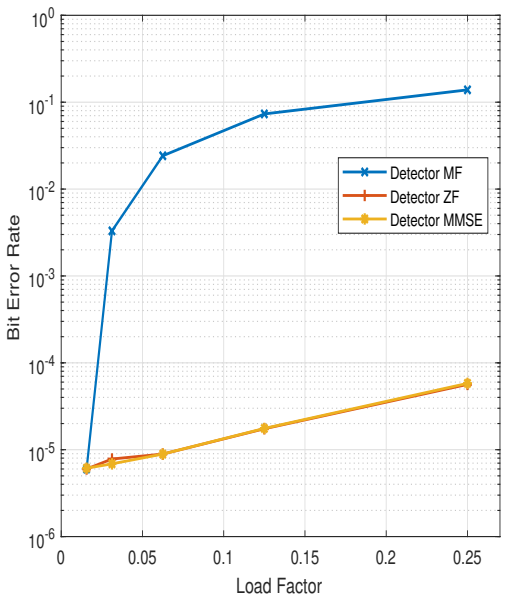

(b)

Source: The authors.

Figure 4 - MCS results for the detectors analysis with imperfect CSI $(\tau=0.2)$, different number of users $(K)$, and number of BS antennas (M): (a) $K=16$ and $M=64$; (b) $K=8$ and $M=128$ and (c) $K=4$ and $M=256$.

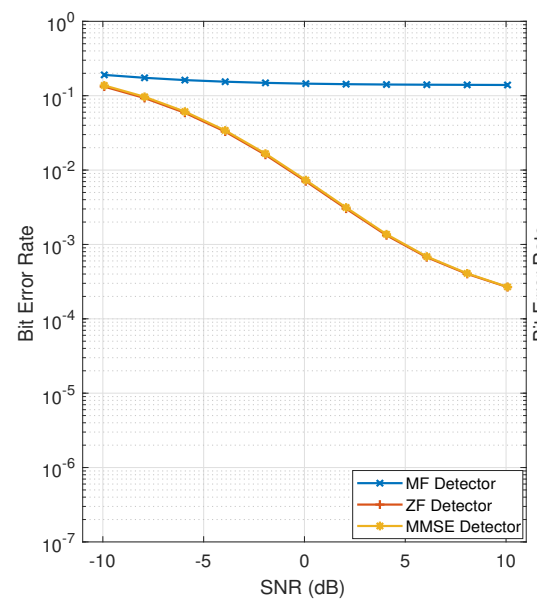

Source: The authors.

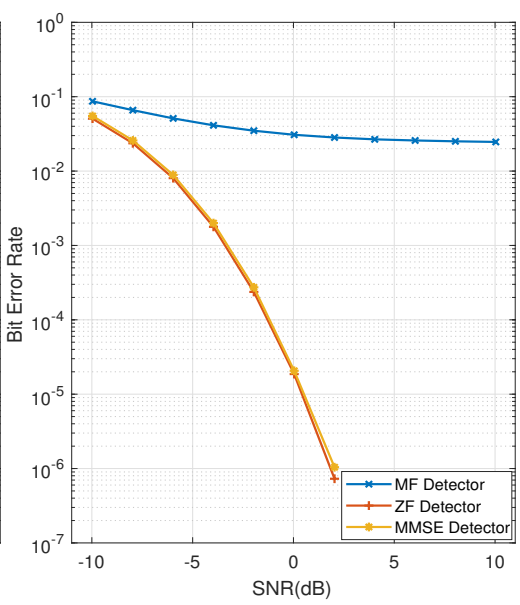

(b)

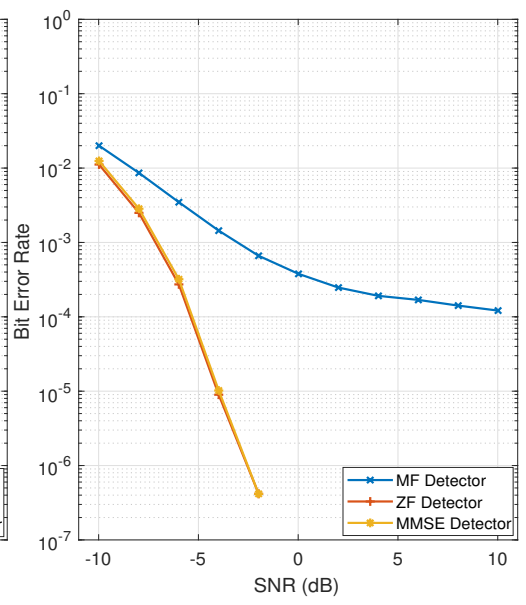

(c) 
Figure 5 - MCS results for the detectors analysis for the average BER $\times$ system loading $\mathscr{L}$, considering imperfect CSI ( $\tau=0.2$ ) and $M=64 \mathrm{BS}$ antennas under low and medium SNR regime: (a) SNR $=-4 \mathrm{~dB}$ and (b) $\mathrm{SNR}=+2 \mathrm{~dB}$.

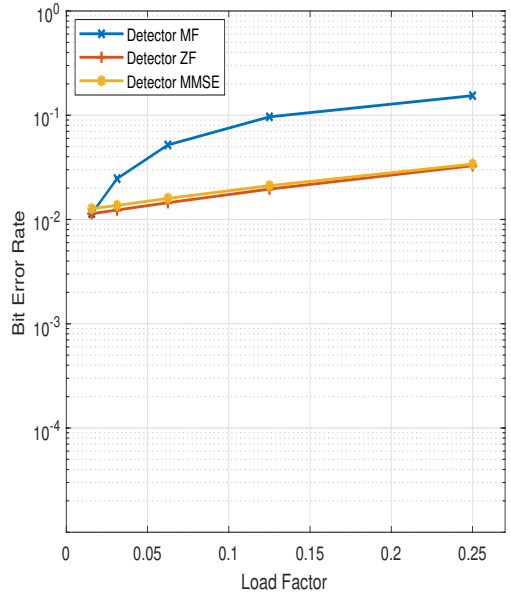

(a)

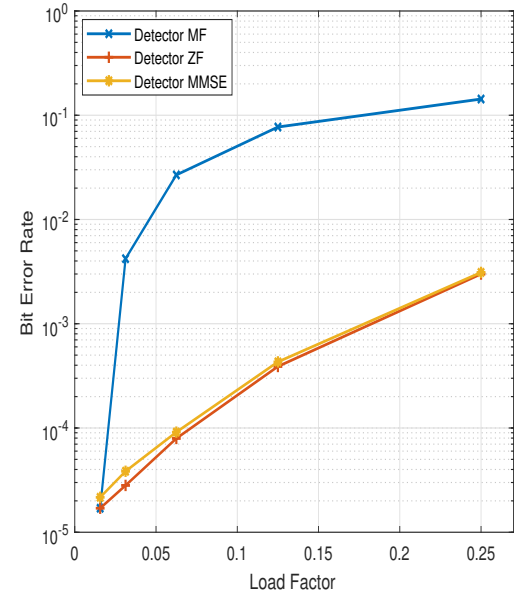

(b)

Source: The authors.

Figure 6 - MCS result for the detectors analysis with imperfect CSI $(\tau=0.4)$, different number of users $(K)$, and number of BS antennas $(M)$ : (a) $K=16$ and $M=64$; (b) $K=8$ and $M=128$ and (c) $K=4$ and $M=256$.

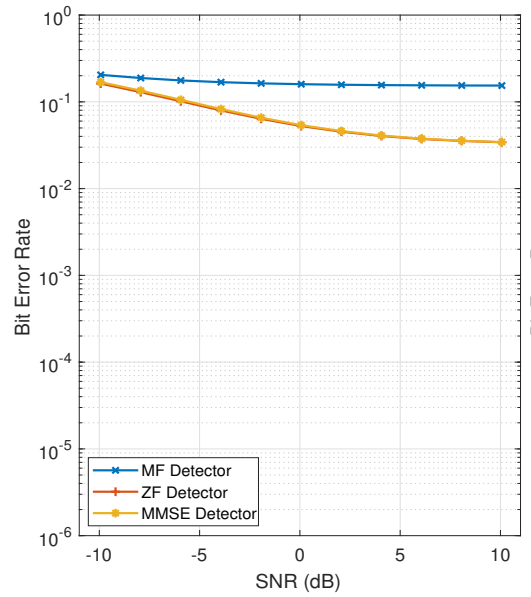

(a)

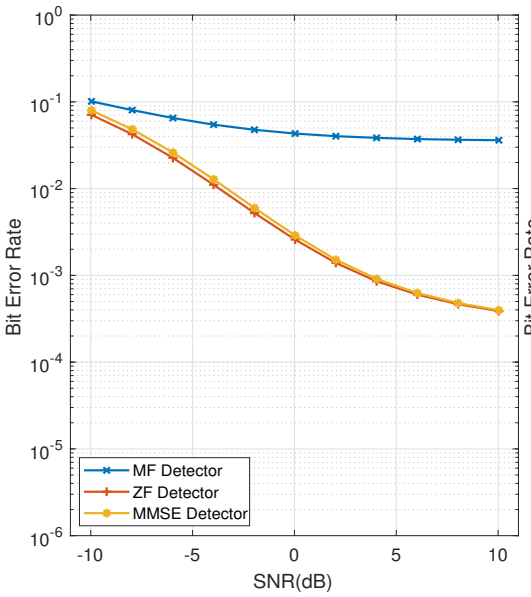

(b)

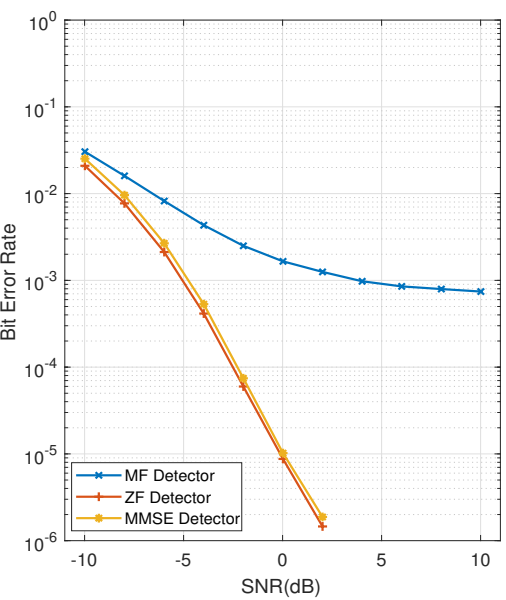

(c)

Source: The authors

Figure 7 - MCS results for the detectors analysis for the average BER $\times$ system loading $\mathscr{L}$, considering imperfect CSI estimates $(\tau=0.4)$, and $M=64 \mathrm{BS}$ antennas under low and medium SNR regime: (a) $\mathrm{SNR}=-4 \mathrm{~dB}$ and (b) $\mathrm{SNR}=+2 \mathrm{~dB}$.

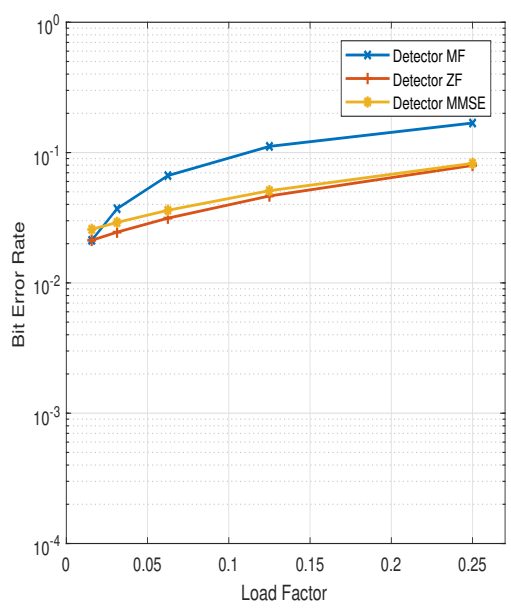

(a)

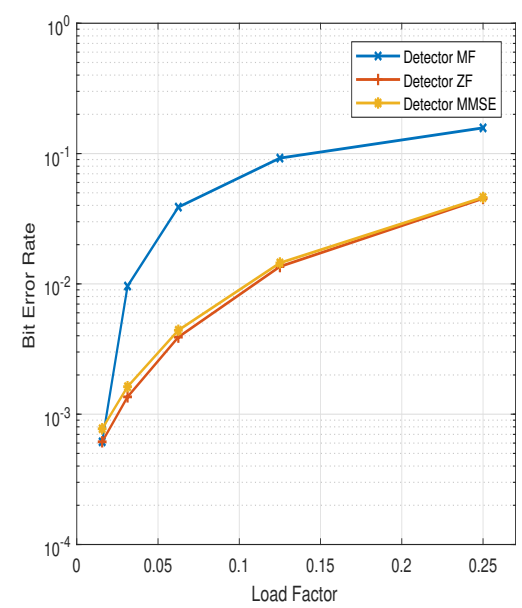

(b) 
Figure 8 - MCS result for the precoders analysis with perfect CSI, different number of users $(K)$, and number of BS antennas $(M)$ : (a) $K=16$ and $M=64$ and (b) $K=8$ and $M=128$.

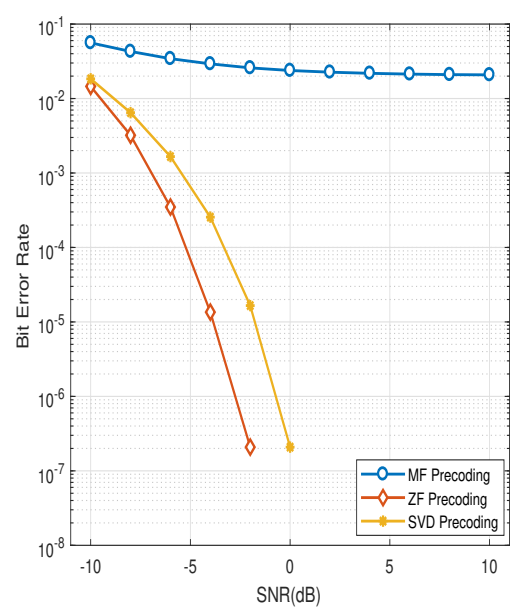

(a)

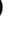

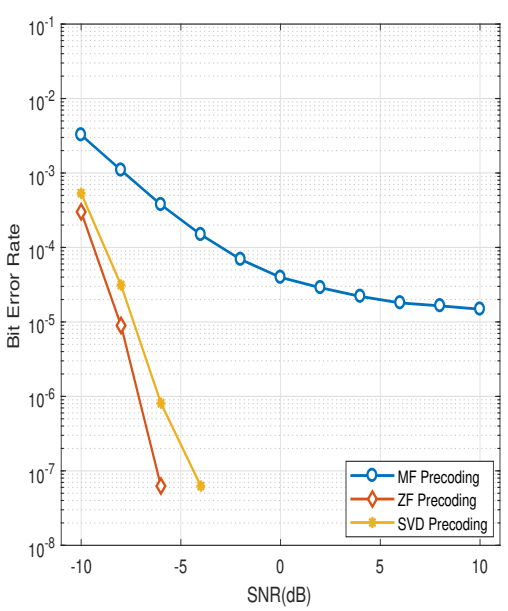

(b)

Source: The authors.

Figure 9 - MCS results for the precoders analysis for the average BER $\times$ system loading $\mathscr{L}$, considering perfect CSI estimates, and $M=64 \mathrm{BS}$ antennas under low SNR regime, where $\mathrm{SNR}=-4 \mathrm{~dB}$.

Source: The authors.

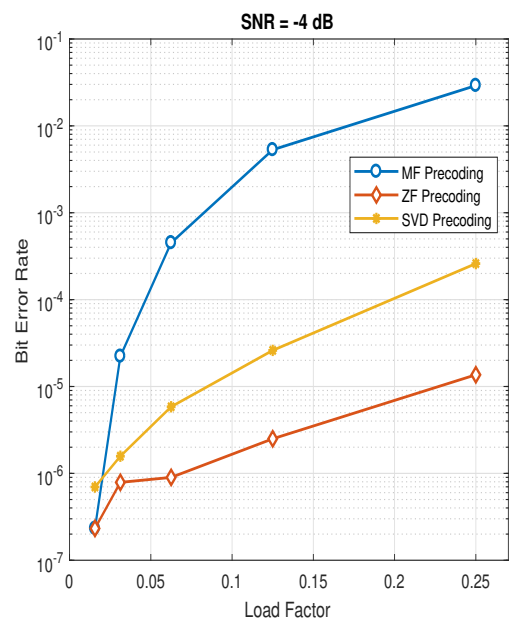

\section{Precoding analysis under CSI errors}

The same channel error estimates model from equation (27) is adopted for this analysis, in which a scenario with a moderate channel estimation inacuracy $(\tau=0.2)$ and another with strong channel errors $(\tau=0.4)$ were selected. From Figures 10-13 it is possible to see how the performance is degraded by the imperfection of the estimates, such degradation is evident in Figure 12(a), with $\tau=0.4$, where, all precoders reach to their respective BER floors, unlike Figure 10 a), where it was not possible to see the BER floors for ZF and SVD yet.
In Figure 12(b) it is also possible to see more errors when compared to Figure 10(b), which uses $\tau=0.2$. However, in more favorable scenarios, such as $\mathscr{L}=0.0156$, optimal results are still obtained, especially in Figures 11(b) and 13(b), with $S N R=+2 d B$. Figures 11(a) and 13(a) may be compared to the perfect CSI case in 9(a), which, even with the same $S N R$, have worse results due to the estimation error. 
Figure 10 - MCS results for the precoders analysis with imperfect CSI $(\tau=0.2)$, different number of users $(K)$, and number of BS antennas (M): (a) $K=16$ and $M=64$ and (b) $K=8$ and $M=128$.

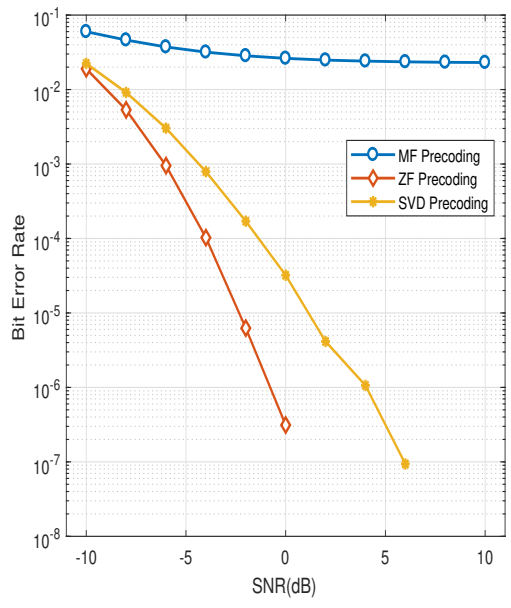

(a)

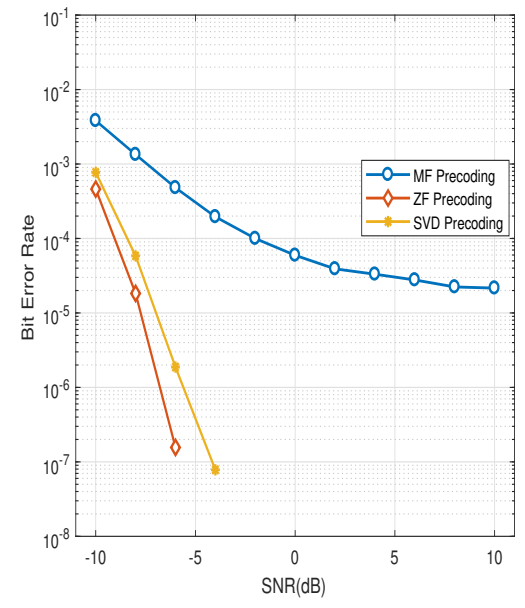

(b)

Figure 11 - MCS results for the precoders analysis for the average BER $\times$ system loading $\mathscr{L}$, considering imperfect CSI estimates $(\tau=0.2$ ), and $M=64 \mathrm{BS}$ antennas under low and medium SNR regime: (a) SNR $=-4 \mathrm{~dB}$ and (b) $\mathrm{SNR}=+2 \mathrm{~dB}$.

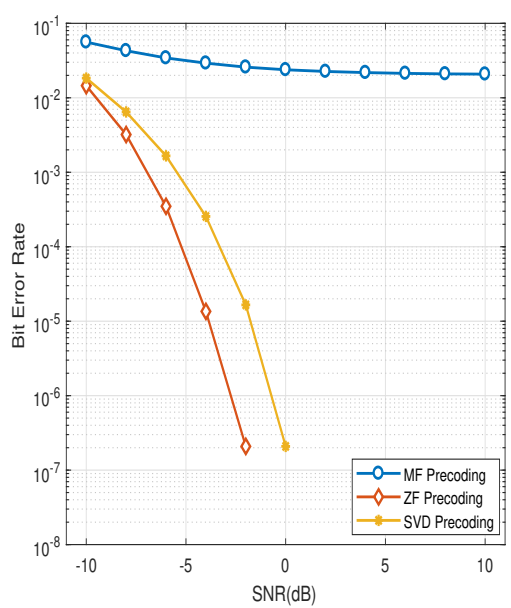

(a)

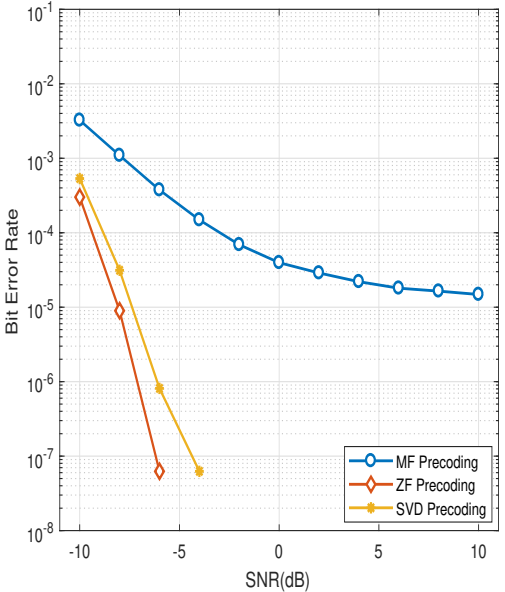

(b)

Source: The authors.

Figure 12 - MCS results for the precoders analysis with imperfect CSI $(\tau=0.4)$, different number of users $(K)$, and number of BS antennas $(M): \mathrm{SNR}=-4 \mathrm{~dB}$ and (b) $\mathrm{SNR}=+2 \mathrm{~dB}$.

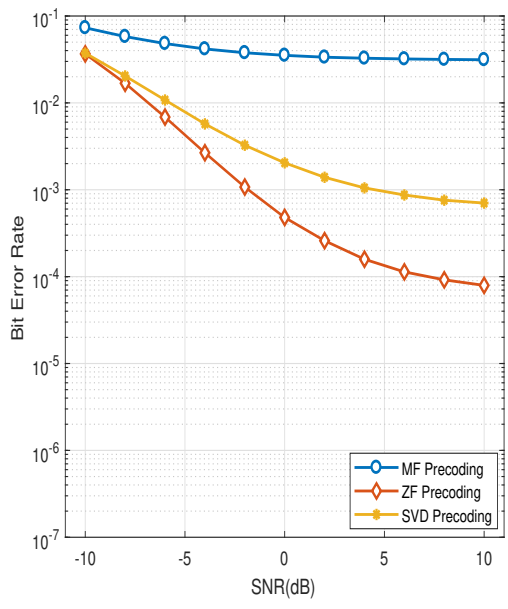

(a)

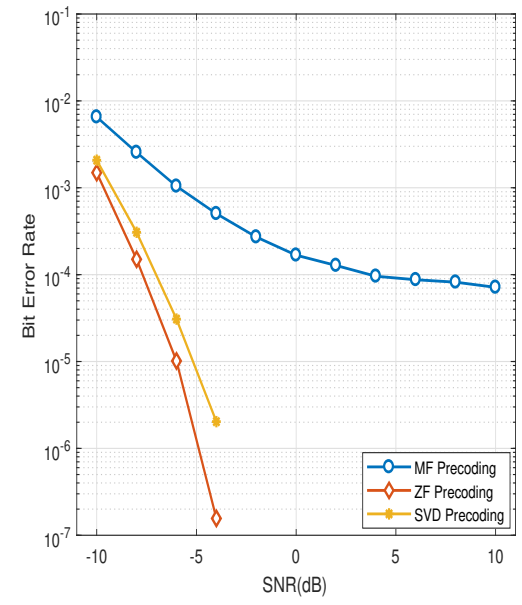

(b) 
Figure 13 - MCS results for the precoders analysis for the average BER $\times$ system loading $\mathscr{L}$, considering imperfect CSI estimates $(\tau=0.4)$, and $M=64 \mathrm{BS}$ antennas under low and medium SNR regime: (a) $\mathrm{SNR}=-4 \mathrm{~dB}$ and (b) $\mathrm{SNR}=+2 \mathrm{~dB}$.

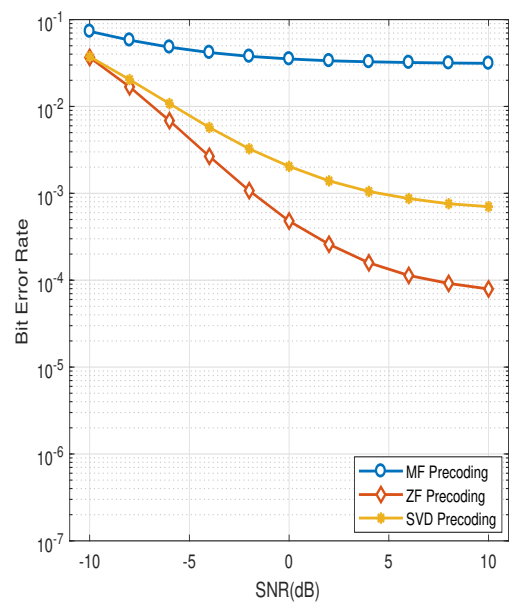

(a)

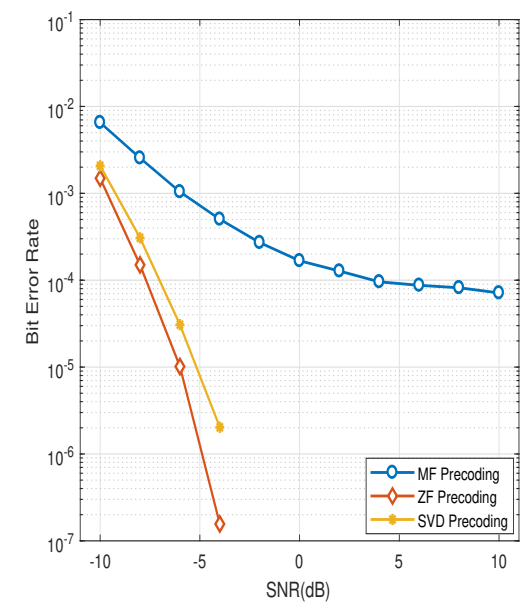

(b)
Source: The authors.

\section{Conclusions}

It has been analyzed the use of linear detectors and precoding methods for massive MIMO systems operating under multiuser scenarios. MCS results indicate that the best performance-complexity trade-off is attainable by the ZF detector since it can zero-forcing the multiuser interference from the other users along the massive number of BS antennas, while it does not require the knowledge of the background noise power, as required by the MMSE.

Regarding the precoding methods herein studied, the ZF precoder achieves the best performance-complexity trade-off results, due to its hability to cancel the interference. However MF and SVD may be used in different scenarios, for the matched-filter case, a good option in simple scenarios, especially when the communication system operating in high SNR and low loading factor $\mathscr{L}$ is allied to low-complexity requirement. The SVD precoding is not feasible in such scenario with single-antenna UEs, since SVD precoding would require precise knowledge of all user's information in each UE, and would be more useful under multiuser multiple antennas in both UEs and BS sides. Hence, in decentralized scenarios, such condition requires precise CSI estimates in all multi-antennas UEs, bringing additional complexity and computational burding in each UE.

Notice that in order to provide a fair comparison, the analyses in this work did not consider power allocation and adaptive modulation and coding methods, although it is known that it increases the entire system performance.
Such strategies may be included in future research considering spectral efficiency and power efficiency performance metrics.

Moreover, the BER performance degradation was analyzed when there are inaccuracies in the channel estimates such analysis, through the model adopted, is not present in the literature, and, therefore, provides novel comparisons with well-known results in the scenario proposed. The complex channel coefficients are estimated with different levels of inaccuracy. Linear ZF and MMSE massive MIMO detectors result in certain robustness against multiuser interference when operating under low and medium system loading and limited level of inaccuracy, specifically, $\tau \leq 0.2$ and $\mathscr{L} \leq 0.25$, respectively. Such robustness is due to the favorable propagation and channel hardening effects that arise in massive MIMO systems.

\section{Acknowledgments}

The authors wish to thank the Laboratory of Telecommunication and Signal Processing (LTSP) of State University of Londrina, and Fundação Araucária, for the financial support.

\section{References}

BJÖRNSON, E.; HOYDIS, J.; SANGUINETTI, L. Massive MIMO has unlimited capacity. IEEE Transactions on Wireless Communications, New York, v. 17, n. 1, p. 574-590, 2018. DOI: 10.1109/TWC.2017.2768423. 
BJÖRNSON, E.; HOYDIS, J.; SANGUINETTI, L. Massive MIMO networks: spectral, energy, and hardware efficiency. Foundations and Trends ${ }^{\circledR}$ in Signal Processing, Netherlands, v. 11, n. 3-4, p. 154-655, 2017. DOI: $10.1561 / 2000000093$.

BOROUJERDI, M. N.; HAGHIGHATSHOAR, S.; CAIRE, G. Low-complexity statistically robust precoder/detector computation for massive MIMO systems. IEEE Transactions on Wireless Communications, New York, v. 17, n. 10, p. 6516-6530, 2018. DOI: 10.1109/TWC.2018.2860951.

BUSCHE, H.; VANAEV, A.; ROHLING, H. SVD-based MIMO precoding and equalization schemes for realistic channel knowledge: design criteria and performance evaluation. Wireless Pers Commun, Dordrecht, v. 48, p. 347-359, 2009. DOI: 10.1007/s11277-008-9526-x.

CHOCKALINGAM, A.; RAJAN, B. S.; Large MIMO systems. Cambridge University Press, Cambridge, p. 1-15, 2014. DOI: 10.1017/CBO9781139208437.

COSTA, B.; MUSSI, A.; ABRAO, T. MIMO detectors under correlated channels. Semina: Ciências Exatas e Tecnológicas, Londrina, v. 37, n. 1, p. 3-12, 2016. DOI: 10.5433/1679-0375.2016v37n1p3.

LIANG, N.; ZHANG, W.; SHEN, C. An uplink interference analysis for massive MIMO systems with MRC and $\mathrm{ZF}$ receivers. IEEE Wireless Communications and Networking Conference (WCNC), Canadá, p. 310-315, 2015. DOI: 10.1109/WCNC.2015.7127488.
MARINELLO, J. C.; ABRAO, T. BER analysis of multicellular MIMO systems with increasing number of bs antennas. ArXiv, Ithaca, p. 1-8, 2014.

MARZETTA, T. L.; LARSSON, E. G.; YANG, H.; NGO, H. Q. Fundamentals of massive MIMO. Cambridge: Cambridge University Press, 2016. DOI: $10.1017 / \mathrm{CBO} 9781316799895$.

MI, D.; DIANATI, M.; ZHANG, L.; MUHAIDAT, S.; TAFAZOLLI, R. Massive MIMO performance with imperfect channel reciprocity and channel estimation error. IEEE Transactions on Communications, New York, v. 65, n. 9, p. 3734-3749, 2017. DOI: 10.1109/TCOMM.2017.2676088.

YOUNAS, T.; LI, J.; ARSHAD, J.; MUNIR, H. M.; TULU, M. M.; YOUNAS, O. Performance of ZF and RZF in massive MIMO with channel aging. IEEE INTERNATIONAL CONFERENCE ON COMPUTER AND COMMUNICATIONS (ICCC), 3., 2017, [s. 1.]. Proceedings [...]. [ S. l.]: IEEE Xplore, 2017. p. 930-934. DOI: 10.1109/CompComm.2017.8322680.

WIESEL, A.; ELDAR, Y. C.; SHAMAI, S. Zero-forcing precoding and generalized inverses. IEEE Transactions on Signal Processing, New York, v. 56, n. 9, p. 4409-4418, 2008. DOI: 10.1109/TSP.2008.924638.

ZU, K. Novel efficient precoding techniques for multiuser MIMO systems. New York: University of York, 2013. 